\title{
Eye Movement Desensitization and Reprocessing in Child and Adolescent Psychology: a Narrative Review
}

Cristina Civilotti ${ }^{1,2,{ }^{*}}$ ๑

Davide Margola ${ }^{3}$

Maria Zaccagnino 4

Martina Cussino ${ }^{4}$

Chiara Callerame 4

Alessia Vicini ${ }^{1}$

Isabel Fernandez ${ }^{5}$

\section{Address}

${ }^{1}$ Department of Psychology, University of Turin, Turin, Italy

${ }^{*}, 2$ Salesian University Institute of Torino Rebaudengo (IUSTO), Turin, Italy

Email: cristina.civilotti@unito.it

${ }^{3}$ Faculty of Psychology, Catholic University of Milan, Milan, Italy

${ }^{4}$ Center of EMDR Therapy for Eating Disorders, Milan, Italy

${ }^{5}$ Centro di Ricerca e Studi in Psicotraumatologia (CRSP), Milan, Italy

Published online: 11 June 2021

(C) The Author(s) 2021

This article is part of the Topical Collection on Early Life Trauma

Keywords EMDR · Psychotherapy · Children · Adolescents

\section{Abstract}

Purpose of review Eye movement desensitization and reprocessing (EMDR) therapy is recognized as an excellent approach to the treatment of trauma in clinical and academic contexts by several national and international organizations. The purpose of this work is to summarize the results of research on EMDR therapy with children and adolescents in various contexts through a narrative review of the scientific literature.

Recent findings Selected studies were clustered in several clinical domains: (1) EMDR and trauma (T), (2) EMDR and violence, (3) EMDR and physical illnesses, and (4) EMDR and psychological problems. Our research indicates how the effectiveness of EMDR therapy within the context of child and adolescent psychology has been widely established in literature. One of the advantages of the use of EMDR therapy with children and adolescents 
is its flexibility, which allows it to be adapted for various age ranges, situations, and cultures.

Summary EMDR therapy is a structured psychotherapeutic method that facilitates the treatment of several psychopathologies and problems related to both traumatic events and more common experiences that are emotionally stressful for children and adolescents, although new studies are necessary to increase its validity.

\section{Introduction}

In recent years, international literature has emphasized the efficacy of eye movement desensitization and reprocessing (EMDR) in the treatment of post-traumatic stress disorder (PTSD). EMDR was first developed by Francine Shapiro [1-3], and today, it can be defined as an empirically supported approach for the treatment of traumatic experiences. A crucial aspect of EMDR therapy seems to be the reprocessing process: It is hypothesized that unpleasant and traumatic events are stored in a dysfunctional way, and EMDR therapy promotes an adaptive processing and resolution of the traumatic experience. The objectives of EMDR are to desensitize the patient to the unpleasant memory, prompt the patient to relive the trauma without experiencing the sensations of imminent threat, and finally, allow them to assimilate and integrate the traumatic event. EMDR psychotherapy is rooted in the adaptive information processing (AIP) model [4], which constitutes the theoretical basis for this clinical approach. To resolve trauma-founded psychological suffering, EMDR is intended to relieve human emotional pain and help people fulfill their full development potential. Its eight-phase approach and three-pronged protocol offer parameters for therapists to organize treatment strategies. Each phase has detailed goals, objectives, and procedural steps.

Before presenting the various EMDR studies carried out on children and adolescents, it is appropriate to specify what childhood trauma means. As in adults, trauma is considered the psychological consequence of a sudden external event presenting a high risk to physical integrity $(\mathrm{T})$ or a series of episodes within traumatic relationships repeated over time and subjectively perceived as dangerous and full of discomfort ( $t$ ). This might generate a sense of helplessness in a child and overwhelm their coping skills, resulting in the deterioration of their emotional and/or cognitive functioning [5, 6]. As in adults, PTSD can develop following exposure to extreme stress situations such as witnessing death, threat or danger of death, serious injury, or sexual assault [7].
Symptoms can include memories and/or unpleasant dreams of the event, psychological suffering, and dissociative and marked physiological reactions in response to triggers that might remind one of the traumatic events. Moreover, avoidance of stimuli associated with trauma as well as alterations in emotions, thoughts, behaviors, and the arousal system can develop. In children, in addition to the above criteria, ritualization and memories related to the trauma can be expressed through play [8-10]. Furthermore, frightening dreams among children (especially the very young) could be connected to the traumatic condition. However, these dreams lack specific content related to the event and are therefore more complicated to examine [11]. In some cases, the child may have reactions following unpleasant flashbacks and behave as if they were reliving the negative event again $[12,13]$. Finally, following a trauma during childhood, delays or changes in development may occur, including in language skills $[14,15]$, depression [16-18], anxiety and separation anxiety $[19,20]$, and specific phobias [21]. If the trauma continues over time and the child lacks the opportunity to build safe attachment relationships, it can generate dysfunctional relational patterns that in turn can lead to a traumatic development characterized by evolutionary fractures and nonadaptive patterns [16]. Several studies [22-26] show that cumulative traumas experienced during childhood are predictive of complex disorders and symptoms in adulthood. In addition, childhood traumatic stress is linked to chronic and/or multiple episodes of sustained abuse [27]. Thus, preventive early intervention is needed to mediate the onset of severe psychopathologies, and EMDR therapy seems to be an appropriate and effective treatment, not only in adulthood but also in childhood and adolescence. Its effectiveness has been described by several authors, such as Muris et al. [28] and Tinker and Wilson [29], from the late nineties until today [30-32]. 


\section{EMDR therapy in children and adolescents}

The standard EMDR protocol can be altered to apply to children and adolescents. For example, language, tools, and some technical aspects have to be modified to suit the cognitive and developmental abilities of minors, including levels of parental involvement. Protocols specific to children and adolescents are manualized in several clinic publications for both individual and group psychotherapeutic approaches [33-36]. In general, the eight phases of the protocol are the following. With phase 1 of EMDR (patient history and treatment planning), the fundamental process of building a therapeutic climate is aligned with the problems of the young patient, respecting their physical and emotional abilities and creating the conditions of safety and stability necessary to process the trauma. At this stage, as well as in subsequent ones, particular emphasis should be placed on assessing the child's age, level of development and understanding, and the context of the child's life experiences by evaluating not only the child's explicit communication but also nonverbal communication (such as changes in posture, tone of voice, or breathing rhythm). Phase 2 (preparation) includes multiple areas forming the basis of the psychotherapeutic path. These include establishing a therapeutic relationship and involving the patient in the clinical process, explaining EMDR in the most appropriate way to the patient and the parental authorities, and determining if and which family members should be included in psychotherapy. Phase 3 (assessment) begins with the identification of the target in continuity with the treatment planning phase. In this phase, targets to work toward in subsequent phases are chosen, defining the image, negative and positive cognitions, emotions, subjective unit of disturbance, and the body sensations associated with the target. Phase 4 (desensitization) involves desensitization to a memory stored in a maladaptive way, leading to an adaptive resolution; for a child, a therapist may need to change the types of bilateral stimulation more often to keep the child engaged, including special objects, toys, drawings, or other therapeutic tools. The goal of phase 5 (installation) is to check the original positive cognition to see if it still works or if another is better, and then install it. The goal of phase 6 (body scan) is to guide the child or adolescent through the steps to obtain a clear body scan without negative sensations. Phase 7 (closure) occurs every time the therapist has to close a session. The goal of the closure phase is to choose an appropriate end point for the session while assisting the patient in the debriefing and stopping any disturbances that may have occurred during the session. Phase 8 (reevaluation) is very important in therapy for children and adolescents, because it is the main way for the therapist to evaluate the success of the reprocessing of information previously stored in a maladaptive way, according to the subjective and developmental goals.

EMDR has been used predominantly as an individual-centered approach to psychotherapy but has been expanded to a group version through several protocols for adults, children, and adolescents, depending on the context. In group clinical work, the therapist offers their technical EMDR skills to support clients' healing processes; moreover, the therapist provides containment and emotional support and assists in achieving and preserving appropriate emotional regulation.

EMDR therapy in national and international guidelines EMDR therapy, to date, has been recognized as an excellent type of therapy for the treatment of PTSD in clinical and academic contexts by several national and international organizations. This approach has been scientifically proven by many randomized controlled trials conducted on traumatized patients and documented in hundreds of publications reporting its effectiveness in the treatment of numerous psychopathologies, including depression, anxiety, phobias, acute mourning, somatic symptoms, and addictions (www.emdr.it). "Like other type of therapies focused on trauma, EMDR aims to reduce the level of subjective stress and strengthen adaptive cognitions related to a traumatic event, but unlike therapies focused on trauma, EMDR does not imply: (a) having to make a detailed description of the event, (b) having a direct discussion of beliefs, (c) carrying out prolonged exposure, or (d) tasks at home" [37]. This can be a great advantage in clinical work with children and adolescents.

EMDR therapy in children is also recommended by the World Health Organization (WHO) [37], the International Society for Traumatic Stress Studies [38], the National Institute for Health and Care Excellence (NICE) [39], and the Child and Adolescent Committee of the European Society on Trauma and Dissociation (ESTD) [40]. The WHO [37] states that both individual and trauma-focused EMDR therapy should be considered for children and adolescents with PTSD when individuals are competent (i.e., trained and supervised) to provide the therapies. NICE guidelines for the treatment of PTSD [39] recommend EMDR therapy for patients aged 7-17 years with a PTSD diagnosis or clinically important symptoms of PTSD presenting more than 3 
months after a traumatic event if they do not respond to or engage with trauma-focused cognitive behavioral therapy (CBT).

Systematic narrative reviews, meta-analyses, and randomized studies [30, 41-45] form a solid base in promoting EMDR therapy and its efficacy to reduce the characteristic symptoms of PTSD in adult, adolescent, and child samples. Usually, the standard protocol is modified to make the treatment easier and more acceptable for children and adolescents with the addition of particular strategies [44] or tools [33].

The purpose of this work is to summarize the results of studies on EMDR therapy with children and adolescents in various contexts.

\section{Research design}

Our objective was to conduct a narrative review of the published scientific literature to evaluate the use of the EMDR approach in children and adolescents. A literature search was carried out about the use of EMDR therapy on children and adolescents. We searched for studies in several databases, including PsycINFO, PubMed, and Google Scholar. The databases were explored with a range of keywords given in different combinations: trauma, EMDR, eye movements, PTSD, child*, adolescent* (an asterisk indicates that the search was not limited to the particular word or fragment). Moreover, we added other studies found in the reference sections of the articles that we evaluated and that had not yet been identified. For the exposition of our results, we opted for a narrative review approach due to the clinical purpose of this work: In a preliminary evaluation of the scientific literature, we found that the interventions, outcomes, and measures of therapeutic effects may vary and are not smoothly comparable between studies, contexts, and clinical conditions. Selection criteria were the theoretical framework used to describe the studies and the research standards they addressed. We made selections to the best of our knowledge and impartiality, but we later acknowledge and discuss possible selection bias. Selected studies were categorized in several main topics, as presented in the following paragraphs.

\section{Results}

\section{EMDR therapy and trauma}

Regarding experiences of extreme stress $(\mathrm{T})$, many studies have focused on mass traumas. In adult populations, several studies have highlighted EMDR protocols' effectiveness for adult populations exposed to trauma via mass disasters and crisis situations in group and individual settings [46-49].

Worldwide, the number of people forced to leave their countries of origin due to war, persecution, conflicts, or other types of violence increased to almost 16.2 million people in 2017, according to estimates. About half of them had not yet reached adulthood [50]. Epidemiological studies confirm that the risk of the onset of psychological problems is high among refugees [51]. The term refugee refers to an individual who has crossed an international border driven by a fear of persecution and who, as a result, asks for protection from another country, which recognizes their asylum request. The clinical histories of these individuals are characterized by repeated exposure to traumatic events (torture, numerous losses of peers and significant figures, serious injuries, war scenarios, 
and food shortage) [52]. Many studies conducted in various geographical areas and contexts have highlighted the use of EMDR protocols with refugee minors [53-58].

Given the critical situation, these people are very likely to be retraumatized and exposed to new traumatic events; the time constraints rooted in the emergency context and the organizational difficulties caused by the socioeconomic characteristics of the context prompt the choice of a group protocol over an individual setting. Most EMDR interventions use the integrative group treatment protocol (EMDR-IGTP) [47] or the integrative group treatment protocol for ongoing traumatic stress (EMDR-IGTP-OTS) [59] with minors belonging to these patient populations. The use of the IGTP-OTS protocol is also attributable to attempts to limit cultural reticence (the sample participants' countries of origin are often not very familiar with practices related to diagnosis and treatment of mental health issues) [55]. In fact, the most important characteristics of EMDR in these contexts are the lack of intrusiveness, the nonnarrative approach with respect to the narrative of the traumatic experience, and the absence of homework assignments between the sessions. With these EMDR protocols, patients are usually not asked to talk about their traumatic memories but to represent them graphically using drawings or symbols. All the studies underline an increase in subjects' psychological well-being associated with a reduction in PTSD, anxiety, and depression symptoms. Results show that this EMDR intervention is effective (data collected after treatment show a decrease in psychological and somatic reactions) and functional in a few group sessions, which makes EMDR a cost-effective treatment.

In parallel, it has been proven that natural disasters (e.g., earthquakes, tsunamis, hurricanes) can cause vulnerability and psychological damage in minors [60]. This context is one of the most common in which EMDR therapy is recognized as an effective treatment for PTSD in children, adolescents, and adults (for a compendium: [61, 62]).

Focusing on children and adolescents, Jayatunge [63] and Fernandez [64] used the standard protocol with samples of minors who had witnessed a tsunami and earthquake, respectively, in response to requests for humanitarian aid from the affected areas. The protocol the Italian EMDR Association used to help children and adolescents after the 2016 earthquake in Central Italy was the EMDR-IGTP [65••]. All results confirmed the effectiveness of this therapy in reducing symptoms associated with PTSD and negative emotions related to mass disasters. They also show how this progress was maintained at the 3month follow-up (in the studies of Fernandez [64] and Maslovaric et al. [65••].

The efficacy of EMDR therapy after mass disasters is extremely well summarized by Brown et al.'s meta-analysis [66], which is based on an investigation of the best techniques for intervention in terms of traumatized adolescents and children. The results, comparing scores obtained before and after treatment, show that EMDR therapy has a significant impact on improving children's psychological conditions.

EMDR therapy is strongly recommended by the California Evidence-Based Clearinghouse for Child Welfare as an evidence-based treatment for children and families involved with the child welfare system (http://www.cebc4cw.org) 
and by the Trauma Institute and Child Trauma Institute (2015).

Child sexual abuse is a common phenomenon, according to a study by Walker et al. [67]: $5.8-34 \%$ of females and $2-11 \%$ of males reported having been sexually abused at some point during childhood. Child sexual abuse is associated with several mental health risk factors, such as substance addiction, suicidal ideation, depression, PTSD, and sexual, relational, and emotional system dysregulation [68-70]. To date, there are very few case studies and clinical practice books about EMDR therapy as a treatment for sexually abused children within the literature [71-73].

Besides the abuse and physical violence children can be subjected to, psychological violence is present as well. Its main characteristic is manifestation through words or acts aimed at forcing the victim to act against their will. This modality makes the presence of danger and trauma in children less obvious, and it is typically the condition that lays the groundwork for the onset of complex and cumulative relational trauma. The EMDR approach is used in a wide variety of sociopolitical and geographical contexts to help children and adolescents recover from the effects of sexual abuse. For example, Jaberghaderi et al. [74] conducted a study on fourteen Iranian girls aged 12-13 years who had been sexually abused. They found that both CBT and EMDR therapy helped the girls recover from the effects of sexual abuse and that structured trauma treatments could be applied to children in Iran. EMDR therapy was significantly more efficient, with large effect sizes for each outcome. In a very different scenario, Farrel et al. [75] recommended the adoption of EMDR treatment for survivors of sexual abuse perpetrated by Roman Catholic priests and other clergy.

The concept of domestic violence includes all acts of physical or sexual abuse, neglect, or other forms of maltreatment that hinder the functional development of an individual [76] and occur within the family. A dysfunctional family climate predisposes children to the development of complex trauma, PTSD, developmental delays, depressive, anxious, or psychosomatic symptoms, internalizing disorders (social withdrawal, self-harming tendencies, substance abuse, excessive complacency), or acting out (aggressive, opposing) and nonadaptive behaviors [77]. A child placed in a context of domestic violence can, in the future, repeat the same dysfunctional relational patterns they learned during childhood [78]. For these reasons, it is vital to break the vicious circle of intergenerational transmission of violent patterns, and many studies have proven EMDR therapy's efficacy in cases of domestic violence on the part of both parents and children [74, 79-81]. All these results support EMDR as an effective treatment modality for survivors of sexual and domestic violence and highlight the need for its inclusion in clinical and supportive programs [82-84]. EMDR therapy is a promising approach, highlighting behavioral improvement in subjects coupled with a reduction in levels of anxiety, depression, and posttraumatic symptoms.

Moving to a slightly different topic, some studies $[85,86]$ illustrate that children with antisocial behaviors have high levels of traumatic exposure. In a study by Soberman et al. [87], 29 boys with conduct problems were randomized into groups receiving standard care or standard care plus three traumafocused EMDR sessions. The EMDR group showed substantial, significant reductions in memory-related distress as well as trends toward reduction of post-traumatic symptoms and a significant reduction of conduct problems at a 
2-month follow-up; the control group showed only slight improvements. The systematic review conducted by Rhoden et al. [88] indicates that EMDR intervention for minors who have committed crimes was the most effective method to reduce post-traumatic symptoms with this sample.

\section{EMDR therapy and physical illnesses}

Physical diseases, especially if they require invasive treatment practices and are disabling or chronic, can traumatize individuals. Several studies have shown that the presence of negative beliefs regarding one's own health, excessive distress, and dysfunctional emotions affect the course of a disease [89]. This creates a vicious circle between psychological and physical factors that could hinder treatment. Some studies [90-92] have also demonstrated the existence of a connection between adverse childhood experiences and adult onset of physical diseases. In this context, interventions that allow one to process traumatic experiences related to disorders are important.

The standard EMDR protocol (with proper modifications to make it more disease-centered) has been used with good results in several pathologies. Verkleij et al. [93] studied changes in the symptomatic manifestation of a teenager with a type of asthma defined as "difficult to treat" or "difficult to control" after a combined treatment of EMDR and CBT. The psychological impact of the disease and the level of asthmatic symptoms after treatment were significantly lower with respect to the initial values.

In the psycho-oncology field, there is evidence $[94,95]$ that EMDR therapy can enhance coping resources in adult patients and reduce post-traumatic and somatic symptoms [96]. In this area, the standard protocol has been altered to focus on the traumatic experience of dealing with a cancer diagnosis [94]. In a 2018 study, Osorio et al. [97] applied an EMDR-IGTP-OTS protocol to a sample of adolescents and young adults with various types of cancer, with the aim of assessing the effectiveness of treatment in reducing post-traumatic symptoms, anxiety, and depression related to their pathology. As hypothesized, the results turned out to be significant in comparison to pre-treatment and the control group, in which no changes were detected. These outcomes were maintained in the follow-up 90 days later. In treating symptoms related to the disease, EMDR therapy was important not only to process the traumatic experience but also to strengthen coping strategies and encourage or restore emotional and relational balance [94].

Recent studies have found that in the pediatric context, medical procedures are potentially traumatic experiences with a range of possible negative psychological consequences; EMDR therapy might be superior to other forms of care in reducing symptoms of blood-injection injury phobia, depression, and sleep problems [98] or assisting young patients who undergo medical treatment and develop subthreshold symptoms of PTSD [99].

\section{EMDR therapy and psychological problems}

Despite the proven effectiveness of EMDR therapy in the treatment of PTSD, evidence suggests that applying this therapy to other psychological disorders produces just as many benefits; we underline that, regarding the ESTD guidelines for the assessment and treatment of children and adolescents with dissociative symptoms and dissociative disorders [40], EMDR therapy is recommended in trauma-processing phases for patients up to 18 years old [100]. 
Taylor and McLachlan [101] point out that the combined effect of EMDR and CBT in a 16-year-old patient diagnosed with obsessive-compulsive disorder helped to decrease the rigidity of her compulsions and obsessions, which were also associated with a history of bullying that contributed to her negative vision of herself and belief that she was worthless. It seemed that there was a connection between the development of obsessive-compulsive symptoms and traumatic exposure [102]. Similar results were obtained in other single case studies on anorexic adolescent patients [103-106]. In this regard, EMDR therapy allowed a broadened vision of the problem and a holistic attitude sympathetic to all aspects related to the girls' traumatic history $[104,107]$. This process then resulted in a quick recovery and the maintenance of results at the follow-up phase.

Regarding childhood phobias, De Roos and de Jongh [108] suggest a possible effect of EMDR therapy on the reduction of phobic symptoms in children and adolescents. These results appear to be in line with those from Rousseau et al. [109], demonstrating how EMDR can increase the learning processes that promotes fear extinction in adults. Some preliminary studies $[110,111]$ have indicated that EMDR therapy could be a promising treatment for substance addiction. It is recognized that exposure to trauma during childhood and adulthood can predispose one to drug or alcohol abuse or related behaviors [112-114]. A 2012 case study reported the successful treatment of Internet addiction in a 13-year-old male through four 45-min sessions based on desensitization of triggers and urge reprocessing protocol [115].

Other research has been conducted on the effectiveness of EMDR in the treatment of major depressive disorders. It is one of the most common psychiatric disorders in childhood and adolescence; according to estimates [116], between 14 and $25 \%$ of young people are subjected to at least one depressive episode before entering adulthood. In a 2019 study by Paauw et al. [93], adolescents diagnosed with major depressive disorder included in EMDR treatment showed a reduction in depressive, somatic, anxiety, and stress-related symptoms (as studies had already shown with adult samples). The social and emotional abilities of these adolescents were also increased. The standard EMDR protocol was used with specific age-related changes; at the end of treatment, $60.9 \%$ of the adolescents no longer met DSM criteria for the diagnosis of major depressive disorder, and 3 months later, during the follow-up, the percentage had risen to $69.8 \%$.

As stated earlier, Soberman et al. [87] also showed improvements in subjects with conduct disorders, maintained at a 2-month follow-up. Wanders et al. [117], on the other hand, illustrated the positive results obtained with EMDR therapy in patients with behavioral and self-esteem problems. This study showed that CBT therapy is also functional in this area but stimulates less significant changes than EMDR.

With regard to intellectual disorders, studies of individuals with deficits in mental and adaptive functioning $[118,119]$ have reported improvements in trauma processing after modified EMDR treatments adapted to the intellectual ages of the participants. Children with intellectual disabilities are more prone to traumatic exposure than peers without this type of disorder [120]. In addition, processing such events is more complicated because of the adaptive and social deficits that they present. This research shows that children with intellectual disabilities actually reprocessed traumatic experiences through EMDR therapy and learned or developed new functional and relational skills. 
Children and adolescents, like adults, can experience traumatic events throughout their lives, but during their growth, difficulties may arise in the emotional, relational, and/or behavioral spheres. The manifestations of psychological distress are not the same as in adults, because the integration of developmental and environmental issues may influence diagnoses in children (especially in very young children). Trauma at the developmental age can assume many faces. Sometimes, people start to exhibit risky behaviors at a very young age, as in the case of refugees, or when parents put children at sociopsychological risk. Sometimes children or adolescents live through medical conditions or major traumas without the capability to fully understand what is going on, or sometimes, their emotional pain arises due to psychological conditions. The effects and consequences that trauma can have on the social and psychological lives of young people are devastating and often co-occur with other psychopathological disorders, such as anxiety, depression, and personality disorders. For this reason, it is extremely important to have effective tools to deal with trauma at the very early stages of development.

The studies presented in this review make a strong contribution to the hypothesis that EMDR appears to be effective for the resolution of trauma and, consequently, relieves the symptoms of anxiety, depression, and stress in children and adolescents with PTSD.

An issue in this area of research is that suffering in childhood can be challenging to be detected because it can be expressed in many different ways-aggressive and oppositional behaviors, anxious states, a tendency to isolate, hyperactivity, school difficulties, sleep disturbances, and etc. Thus, there is a potential for difficulty in clinical work and research, as a clearly defined diagnosis is not always possible; moreover, psychotherapists and researchers should use appropriate and standardized measures to assess children because the use of unknown or personal measures can affect a study's conclusions and acceptability in the scientific community.

All the studies that we included combine to indicate how important it is for minors to have a space where they feel welcome and understood and can make sense of their suffering, emotions, and behaviors. Several EMDR therapy protocols, with appropriate adaptations, help younger patients express emotional discomfort, gain greater knowledge of their abilities and potential, accept their limits, and reduce their psycho-emotional suffering through improved management of their own emotional states.

On a clinical level, as indicated by Ahmad et al. [44] and by Civilotti et al. [121], it is important to consider the capacity to recognize positive and negative cognitions and emotions rooted in the development stage and the sociocultural context of the child or adolescent. Some deviations from the standard EMDR protocol are necessary in recalling traumatic events, for example, when identifying negative cognitions and emotions and especially in the identification of positive cognitions. Sometimes, it is important to include caregivers in the narrative or during the elaboration process [122]. 


\section{Limitations}

\section{Conclusions}

As with all narrative reviews, this work has some limitations, as described in several papers on this subject [123, 124]. It provides an experiential and explicit perspective on the use of EMDR therapy in childhood and adolescence psychology, reflecting a clinical perspective rather than a more neutral academic view. Instead of using a systematic narrative review approach based on the findings of comprehensive and systematic literature searches of all available resources and with a great minimization of selection bias, we decided to use a narrative approach. This method mirrors the clinical reality in which the objectivity of a rigorous research design is not always possible because of several causes-for example, ethical reasons (e.g., the impossibility of delay or not treating some patients), complexity (e.g., the difficulty to compare different subjects and their intrinsic, individual differences), and methodological reasons (e.g., it may be hard to compare clinical approaches or treatment without considering variables such as preexisting conditions, therapeutic alliance).

In conclusion, the effectiveness of EMDR therapy in children and adolescents has been widely shown in literature, although new studies with larger samples are desirable to increase its validity.

In fact, many studies have been done on individual clinical cases or very small samples. Randomization was often not possible due to the intrinsic specificities of the subjects and their traumas (e.g., children living in areas affected by natural disasters). One advantage of the use of EMDR therapy with children and adolescents is its flexibility, which allows a therapist to adapt it to a patient's age and culture; another strength is its applicability in a group setting, even after mass disasters, often characterized by a high demand for help and low availability of resources. In these cases, early psychological support can act as a protective factor against the onset of more serious conditions.

\section{Data Availability}

Not applicable

\section{Code Availability}

Not applicable

\section{Funding}

Open access funding provided by Università degli Studi di Torino within the CRUI-CARE Agreement. 


\section{Declarations}

\section{Conflict of Interest}

Cristina Civilotti declares that she has no conflict of interest. Davide Margola declares that he has no conflict of interest. Maria Zaccagnino declares that she has no conflict of interest. Martina Cussino declares that she has no conflict of interest. Chiara Callerame declares that she has no conflict of interest. Alessia Vicini declares that she has no conflict of interest. Isabel Fernandez declares that she has no conflict of interest.

Open Access This article is licensed under a Creative Commons Attribution 4.0 International License, which permits use, sharing, adaptation, distribution and reproduction in any medium or format, as long as you give appropriate credit to the original author(s) and the source, provide a link to the Creative Commons licence, and indicate if changes were made. The images or other third party material in this article are included in the article's Creative Commons licence, unless indicated otherwise in a credit line to the material. If material is not included in the article's Creative Commons licence and your intended use is not permitted by statutory regulation or exceeds the permitted use, you will need to obtain permission directly from the copyright holder. To view a copy of this licence, visit http://creativecommons.org/licenses/by/4.0/.

\section{References}

Papers of particular interest, published recently, have been highlighted as:

$\bullet \quad$ Of major importance

1 Shapiro F. Eye movement desensitization: a new treatment for post-traumatic stress disorder. J Behav Ther Exp Psychiatry. 1989;20(3):211-17.

2 Shapiro F. Efficacy of the eye movement desensitization procedure in the treatment of traumatic memories. J Trauma Stress. 1989;2(2):199-223.

3 Shapiro F. Eye movement desensitization and reprocessing (EMDR) therapy: basic principles, protocols, and procedures. Guilford Publications; 2017.

4 Solomon RM, Shapiro F. EMDR and the adaptive information processing model potential mechanisms of change. J EMDR Pract Res. 2008;2(4):315-25.

5 Pillay AL, Schoubben-Hesk S. Depression, anxiety, and hopelessness in sexually abused adolescent girls. Psychol Rep. 2001;88(3):727-33.

6 Perry BD, Azad I. Posttraumatic stress disorders in children and adolescents. Curr Opin Pediatr. 1999;11(4):310-16.

7 Association AP. Diagnostic and statistical manual of mental disorders (DSM-5 ${ }^{\circledR}$ ). American Psychiatric Pub; 2013.

8 Norton B, Ferriegel M, Norton C. Somatic expressions of trauma in experiential play therapy. Int J Play Ther. 2011;20(3):138-52.

9 Ogawa Y. Childhood trauma and play therapy intervention for traumatized children. J Prof Couns Pract Theory Res. 2004;32(1):19-29.
White M. Children, trauma and subordinate storyline development. Int J Narrat Ther Commun Work. 2005;(3/4):143-65.

11 Scheeringa MS, Zeanah CH. Symptom expression and trauma variables in children under 48 months of age. Infant Ment Health J. 1995;16(4):259-70.

12 Shapiro LR. Memory and child abuse. Encycl Women Crime. 2019;1-8.

13 Thompson B. Trauma and attachment. Child Youth Ment Heal Canada Cases from Front Settings. 2018;40.

14 Kinniburgh KJ, Blaustein M, Spinazzola J, Van der Kolk BA. Attachment, Self-Regulation, and Competency: a comprehensive intervention framework for children with complex trauma. Psychiatr Ann. 2017;35(5):42430 .

15 Acquadro Maran D, Soro G, Biancetti A, Zanotta T. Serving others and gaining experience: a study of university students participation in service learning. High Educ Q. 2009;63(1):46-63.

16 Cook A, Spinazzola J, Ford J, Lanktree C, Blaustein M, Cloitre $\mathrm{M}$, et al. Complex trauma in children and adolescents. Psychiatr Ann. 2017;35(5):390-98.

17 Van Nierop M, Viechtbauer W, Gunther N, Van Zelst C, De Graaf R, Ten Have $M$, et al. Childhood trauma is associated with a specific admixture of affective, anxiety, and psychosis symptoms cutting across traditional diagnostic boundaries. Psychol Med. 2015;1-12.

18 De Haan A, Landolt MA, Fried EI, Kleinke K, Alisic E, Bryant R, et al. Dysfunctional posttraumatic cognitions, 
posttraumatic stress and depression in children and adolescents exposed to trauma: a network analysis. J Child Psychol Psychiatry. 2020;61(1):77-87.

19 Emre O, Ulutas A, Inci R, Cosanay B. The relationship between stress response after trauma with anxiety and depression levels of Syrian children. Medicine (Baltimore). 2020;9(1):164-69.

20 El Baba R, Colucci E. Post-traumatic stress disorders, depression, and anxiety in unaccompanied refugee minors exposed to war-related trauma: a systematic review. Int J Cult Ment Health. 2018;11(2):194-207.

21 McCloskey LA, Walker M. Posttraumatic stress in children exposed to family violence and single-event trauma. J Am Acad Child Adolesc Psychiatry. 2000;39(1):108-15.

22 Cloitre M, Stolbach BC, Herman JL, van der Kolk B, Pynoos R, Wang J, et al. A developmental approach to complex PTSD: Childhood and adult cumulative trauma as predictors of symptom complexity. J Trauma Stress. 2009;22(5):399-408.

23 Sölva K, Haselgruber A, Lueger-Schuster B. The relationship between cumulative traumatic experiences and ICD-11 post-traumatic symptoms in children and adolescents in foster care: the mediating effect of sense of coherence. Child Abuse Negl. 2020;101:104388.

24 Turner RJ, Lloyd DA. Lifetime traumas and mental health: the significance of cumulative adversity. J Health Soc Behav. 1995;360-76.

25 Shevlin M, Houston JE, Dorahy MJ, Adamson G. Cumulative traumas and psychosis: an analysis of the national comorbidity survey and the British Psychiatric Morbidity Survey. Schizophr Bull. 2008;34(1):193-99.

26 Briere J, Agee E, Dietrich A. Cumulative trauma and current posttraumatic stress disorder status in general population and inmate samples. Psychol Trauma Theory Res Pract Policy. 2016;8(4):439-46.

27 Felitti VJ, Anda RF, Nordenberg D, Williamson DF, Spitz AM, Edwards V, et al. Relationship of childhood abuse and household dysfunction to many of the leading causes of death in adults: the Adverse Childhood Experiences (ACE) Study. Am J Prev Med. 1998;14(4):245-58.

28 Muris P, Merckelbach H, Holdrinet I, Sijsenaar M. Treating phobic children: effects of EMDR versus exposure. J Consult Clin Psychol. 1998;66(1):193-98.

29 Tinker RH, Wilson SA. Through the eyes of a child: EMDR with children. WW Norton \& Co; 1999.

$30 \bullet$ Adler-Tapia R, Settle C. Evidence of the efficacy of EMDR with children and adolescents in individual psychotherapy: a review of the research published in peer-reviewed journals. J EMDR Pract Res. 2009;3(4):232-47.

This work consists of a very accurate literature review regarding the application of the EMDR protocol in the individual setting in developmental age.

31 Yunitri N, Kao C-C, Chu H, Voss J, Chiu H-L, Liu D, et al. The effectiveness of eye movement desensitization and reprocessing toward anxiety disorder: a metaanalysis of randomized controlled trials. J Psychiatr Res. 2020;123:102-13.
32 Beer R. Efficacy of EMDR therapy for children with PTSD: a review of the literature. J EMDR Pract Res. 2018;12(4):177-95.

33 Adler-Tapia R, Settle C. EMDR and the art of psychotherapy with children: treatment manual. Springer Publishing Company; 2008.

34 Gomez AM. EMDR therapy and adjunct approaches with children: complex trauma, attachment, and dissociation. Springer Publishing Company; 2012. Adler-Tapia R, Settle C. EMDR and the art of psychotherapy with children: infants to adolescents. Springer Publishing Company; 2016.

36 Maslovaric G. EMDR di gruppo. Insieme verso il BenEssere, Protocolli di Intervento. Roma: ApertaMenteWeb; 2020.

WHO. Guidelines for the management of conditions specifically related to stress. World Health Organization; 2013.

International Society for Traumatic Stress Studies. No Title. 2018. p. https://istss.org/home. Accessed 15 Nov 2020.

39 NICE. No Title. 2018. p. www.nice.org.uk/guidance/ ng116, Accessed 15 Nov 2020. NICE Nice Guidelin.

40 ESTD. Child and Adolescent Committee of the European Society on Trauma and Dissociation www.estd. org/content/estd-child-and-adolescent-committee. Accessed 15 Nov 2020. p. Child and Adolescent Committee of the European Soc.

41 Barron IG, Bourgaize C, Lempertz D, Swinden C, Darker-Smith S. Eye movement desensitization reprocessing for children and adolescents with posttraumatic stress disorder: a systematic narrative review. J EMDR Pract Res. 2019;13(4):270-83.

42 Mavranezouli I, Megnin-Viggars O, Trickey D, MeiserStedman R, Daly C, Dias S, et al. Cost-effectiveness of psychological interventions for children and young people with post-traumatic stress disorder. J Child Psychol Psychiatry. 2020;61(6):699-710.

43 Diehle J, Opmeer BC, Boer F, Mannarino AP, Lindauer RJL. Trauma-focused cognitive behavioral therapy or eye movement desensitization and reprocessing: what works in children with posttraumatic stress symptoms? A randomized controlled trial. Eur Child Adolesc Psychiatry. 2015;24(2):227-36.

44 Ahmad A, Sundelin-Wahlsten V. Applying EMDR on children with PTSD. Eur Child Adolesc Psychiatry. 2008;17(3):127-32.

Rodenburg R, Benjamin A, de Roos C, Meijer AM, Stams GJ. Efficacy of EMDR in children: a meta-analysis. Clin Psychol Rev. 2009;29(7):599-606.

46 Jarero I, Artigas L. The EMDR integrative group treatment protocol: application with adults during ongoing geopolitical crisis. J EMDR Pract Res. 2010;4(4):14855.

47 Jarero I, Artigas L. The EMDR Integrative Group Treatment Protocol: EMDR group treatment for early intervention following critical incidents. Eur Rev Appl Psychol. 2012;62(4):219-22. 
48 Silver SM, Rogers S, Knipe J, Colelli G. EMDR therapy following the 9/11 terrorist attacks: a community-based intervention project in New York City. Int J Stress Manag. 2005;12(1):29-42.

49 Jarero I, Artigas L, Luber M. The EMDR protocol for recent critical incidents: application in a disaster mental health continuum of care context. J EMDR Pract Res. 2011;5(3):82-94.

50 UNHCR. No Title. Global Trends Force Displacement in 2017 Retrieved at https://www.unhcr.org/ globaltrends2017/. Accessed 15 Nov 2020. 2018

51 Fazel M, Wheeler J, Danesh J. Prevalence of serious mental disorder in 7000 refugees resettled in western countries: a systematic review. Lancet.

2005;365(9467):1309-14.

52 Goodwin-Gill GS, Mc Adam J. The refugee in international law. Oxford: Oxford University Press; 2007.

53 Oras R, de Ezpeleta SC, Ahmad A. Treatment of traumatized refugee children with eye movement desensitization and reprocessing in a psychodynamic context. Nord J Psychiatry. 2004;58(3):199-203.

54 Molero RJ, Jarero I, Givaudan M. Longitudinal multisite randomized controlled trial on the provision of the EMDR-IGTP-OTS to refugee minors in Valencia, Spain. Am J Appl Psychol. 2019;8(4):77-88.

55 Perilli S, Giuliani A, Pagani M, Mazzoni GP, Maslovaric G, Maccarrone B, et al. EMDR group treatment of children refugees-a field study. J EMDR Pract Res. 2019;13(2):143-55.

Smyth-Dent KL, Fitzgerald J, Hagos Y. A field study on the EMDR integrative group treatment protocol for ongoing traumatic stress provided to adolescent Eritrean refugees living in Ethiopia. Psychol Behav Sci Int J. 2019;12(4):1-12.

57 Hurn R, Barron I. The EMDR integrative group treatment protocol in a psychosocial program for refugee children: a qualitative pilot study. J EMDR Pract Res. 2018;12(4):208-23.

58 Wadaa NN, Zaharim NM, Alqashan HF. The use of EMDR in treatment of traumatized Iraqi children. Dig Middle East Stud. 2010;19(1):26-36.

59 Jarero I, Artigas L. AIP model-based acute trauma and ongoing traumatic stress theoretical conceptualization. Iberoam J Psychotraumatol Dissoc. 2018;10(1):1-7.

60 Wang C-W, Chan CLW, Ho RTH. Prevalence and trajectory of psychopathology among child and adolescent survivors of disasters: a systematic review of epidemiological studies across 1987-2011. Soc Psychiatry Psychiatr Epidemiol. 2013;48(11):1697-720.

61 Maxfield L. EMDR treatment of recent events and community disasters. Springer; 2008.

62 Shapiro E, Maxfield L. The efficacy of EMDR early interventions. J EMDR Pract Res. 2019;13(4):291-301.

63 Jayatunge RM. Combating tsunami disaster through EMDR. J EMDR Pract Res. 2008;2(2):140-45.

64 Fernandez I. EMDR as treatment of post-traumatic reactions: a field study on child victims of an earthquake. Educ Child Psychol. 2007;24(1):65-72.
65•• Maslovaric G, Zaccagnino M, Mezzaluna C, Perilli S, Trivellato D, Longo $\mathrm{V}$, et al. The effectiveness of eye movement desensitization and reprocessing integrative group protocol with adolescent survivors of the Central Italy earthquake. Front Psychol. 2017;8:18-26.

This scientific article reports an example of EMDR group interventions in children and adolescents after a natural disaster.

66 Brown RC, Witt A, Fegert JM, Keller F, Rassenhofer M, Plener PL. Psychosocial interventions for children and adolescents after man-made and natural disasters: a meta-analysis and systematic review. Psychol Med. 2017;47(11):1893-905.

67 Walker JL, Carey PD, Mohr N, Stein DJ, Seedat S. Gender differences in the prevalence of childhood sexual abuse and in the development of pediatric PTSD. Arch Women's Ment Heal. 2004;7(2):111-21.

68 Dvir Y, Ford JD, Hill M, Frazier JA. Childhood maltreatment, emotional dysregulation, and psychiatric comorbidities. Harv Rev Psychiatry. 2014;22(3):149_ 61.

69 Ford JD, Hartman JK, Hawke J, Chapman JF. Traumatic victimization, posttraumatic stress disorder, suicidal ideation, and substance abuse risk among juvenile justice-involved youth. J Child Adolesc Trauma. 2008;1(1):75-92.

70 Margola D, Donato S, Accordini M, Emery RE, Snyder DK. Dyadic coping in couple therapy process: an exploratory study. Fam Process. 2018;57(2):324-41.

71 Jaberghaderi N, Greenwald R, Rubin A, Zand SO, Dolatabadi S. A comparison of CBT and EMDR for sexually-abused Iranian girls. Clin Psychol Psychother An Int J Theory Pract. 2004;11(5):358-68.

72 Adler-Tapia R, Settle C, Shapiro F. Eye movement desensitization and reprocessing (EMDR) psychotherapy with children who have experienced sexual abuse and trauma. Handb Child Sex Abus Identification, Assessment, Treat 2012;229-50.

73 Swinden C. The child-centered EMDR approach: a case study investigating a young girl's treatment for sexual abuse. J EMDR Pract Res. 2018;12(4):282-96.

74 Jaberghaderi N, Rezaei M, Kolivand M, Shokoohi A. Effectiveness of cognitive behavioral therapy and eye movement desensitization and reprocessing in child victims of domestic violence. Iran J Psychiatry. 2019;14(1):67-75.

75 Farrell D, Dworkin M, Keenan P, Spierings J. Using EMDR with survivors of sexual abuse perpetrated by Roman Catholic priests. J EMDR Pract Res. 2010;4(3):124-33.

76 Carlson BE, Worden AP. Attitudes and beliefs about domestic violence: results of a public opinion survey: I. Definitions of domestic violence, criminal domestic violence, and prevalence. J Interpers Violence. 2005;20(10):1197-218.

77 Holmes MR. The sleeper effect of intimate partner violence exposure: long-term consequences on young children's aggressive behavior. J Child Psychol Psychiatry. 2013;54(9):986-95. 
78 Ehrensaft MK, Cohen P, Brown J, Smailes E, Chen H, Johnson JG. Intergenerational transmission of partner violence: a 20-year prospective study. J Consult Clin Psychol. 2003;71(4):741-53.

79 Stowasser JE. EMDR and family therapy in the treatment of domestic violence. Handb EMDR Fam Ther Process. 2007;243.

80 Ricci RJ, Clayton CA, Shapiro F. Some effects of EMDR on previously abused child molesters: theoretical reviews and preliminary findings. J Forens Psychiatry Psychol. 2006;17(4):538-62.

81 Jiménez G, Becker Y, Varela C, García P, Nuño MA, Pérez MC, et al. Multicenter randomized controlled trial on the provision of the EMDR-PRECI to female minors victims of sexual and/or physical violence and related PTSD diagnosis. Am J Appl Psychol. 2020;9(2):42-51.

82 Begotti T, Acquadro Maran D. Characteristics of cyberstalking behavior, consequences, and coping strategies: a cross-sectional study in a sample of Italian university students. Future Internet. 2019;11(5):120.

83 Civilotti C, Sciascia C, Zaccagnino M, Varetto A, Acquadro Maran D. States of mind with respect to adult attachment and reflective functioning in a sample of men detained for stalking: evaluation and clinical implications. SAGE Open. 2020;10(4).

84 Acquadro Maran D, Varetto A. Psychological impact of stalking on male and female health care professional victims of stalking and domestic violence. Front Psychol. 2018;9:321.

85 Baglivio MT, Wolff KT, DeLisi M, Jackowski K. The role of adverse childhood experiences (ACEs) and psychopathic features on juvenile offending criminal careers to age 18 . Youth Violence Juv Justice. 2020;18(4):337-64.

86 Dierkhising CB, Sánchez JA, Gutierrez L. "It changed my life": traumatic loss, behavioral health, and turning points among gang-involved and justice-involved youth. J Interpers Violence. 2019;0886260519847779. Soberman GB, Greenwald R, Rule DL. A controlled study of eye movement desensitization and reprocessing (EMDR) for boys with conduct problem. J Aggress Maltreat Trauma. 2002;6(1):217-36.

88 Rhoden M-A, Macgowan MJ, Huang H. A systematic review of psychological trauma interventions for juvenile offenders. Res Soc Work Pract. 2019;29(8):892-909.

89 Civilotti C, Acquadro Maran D, Santagata F, Varetto A, Stanizzo MR. The use of the Distress Thermometer and the Hospital Anxiety and Depression Scale for screening of anxiety and depression in Italian women newly diagnosed with breast cancer. Support Care Cancer. 2020;

90 Wegman HL, Stetler C. A meta-analytic review of the effects of childhood abuse on medical outcomes in adulthood. Psychosom Med. 2009;71(8):805-12.

91 Brown MJ, Thacker LR, Cohen SA. Association between adverse childhood experiences and diagnosis of cancer. PLoS One. 2013;8(6):e65524.

92 Fuller-Thomson E, Brennenstuhl S. Making a link between childhood physical abuse and cancer: results from a regional representative survey. Cancer
Interdiscip Int J Am Cancer Soc. 2009;115(14):334150.

93 Verkleij M, Beelen A, van Ewijk BE, Geenen R. Multidisciplinary treatment in children with problematic severe asthma: a prospective evaluation. Pediatr Pulmonol. 2017;52(5):588-97.

94 Faretta E, Borsato T, Civilotti C, Fernandez I, Pagani M. EMDR and CBT: a comparative clinical study with oncological patients. J EMDR Pract Res. 2016;10(3).

95 Faretta E, Civilotti C. EMDR therapy in psycho-oncology: a bridge between mind and body. J EMDR Pract Res. 2016;10(3).

96 Civilotti C, Castelli L, Binaschi L, Cussino M, Tesio V, Fini GD, et al. Dissociative symptomatology in cancer patients. Front Psychol. 2015;6(FEB).

97 Osorio A, Pérez MC, Tirado SG, Jarero I, Givaudan M. Randomized controlled trial on the EMDR integrative group treatment protocol for ongoing traumatic stress with adolescents and young adults patients with cancer. Am J Appl Psychol. 2018;7(4):50-6.

98 Meentken MG, van der Mheen $M$, van Beynum IM, Aendekerk EWC, Legerstee JS, van der Ende J, et al. EMDR for children with medically related subthreshold PTSD: short-term effects on PTSD, blood-injectioninjury phobia, depression and sleep. Eur J

Psychotraumatol. 2020;11(1):1705598.

99 Meentken MG, van Beynum IM, Aendekerk EWC, Legerstee JS, El Marroun H, van der Ende J, et al. Eye movement desensitization and reprocessing (EMDR) in children and adolescents with subthreshold PTSD after medically related trauma: design of a randomized controlled trial. Eur J Psychotraumatol.

2018;9(1):1536287.

100 Karadag M, Gokcen C, Sarp AS. EMDR therapy in children and adolescents who have post-traumatic stress disorder: a six-week follow-up study. Int J Psychiatry Clin Pract. 2020;24(1):77-82.

101 Taylor A, McLachlan NH. Treating a 16 year old with a history of severe bullying: supplementing cognitive behavioural therapy with EMDR within the context of a case formulation approach. J Child Adolesc Trauma. 2019;12(4):561-70.

102 de Silva P, Marks M. The role of traumatic experiences in the genesis of obsessive-compulsive disorder. Behav Res Ther. 1999;37(10):941-51.

103 Zaccagnino M, Cussino M, Callerame C, Civilotti C, Fernandez I. Anorexia nervosa and EMDR: a clinical case. J EMDR Pract Res. 2018;12(4).

104 Civilotti C, Cussino M, Callerame C, Fernandez I, Zaccagnino M. Changing the adult state of mind with respect to attachment: an exploratory study of the role of EMDR psychotherapy. J EMDR Pract Res. 2019;13(3).

105 Balbo M, Zaccagnino M, Cussino M, Civilotti C. Eye movement desensitization and reprocessing (EMDR) and eating disorders: a systematic review. Clin Neuropsychiatry. 2017;14(5).

106 Smajić VA, de la Fosse C. EMDR treatment for anorexia nervosa triggered by early traumatic experiences. 
Psychother Achiev Heal Well-being Child Young People. 2019;2(2):65-76.

107 Zaccagnino M, Cussino M, Callerame C, Civilotti C, Fernandez I. Anorexia nervosa and EMDR: a clinical case. J EMDR Pract Res. 2017;11(1).

108 De Roos C, de Jongh A. EMDR treatment of children and adolescents with a choking phobia. J EMDR Pract Res. 2008;2(3):201-11.

109 Rousseau PF, El Khoury-Malhame M, Reynaud E, Zendjidjian X, Samuelian JC, Khalfa S. Neurobiological correlates of EMDR therapy effect in PTSD. Eur J Trauma Dissoc. 2019;3(2):103-11.

110 Perez-Dandieu B, Tapia G. Treating trauma in addiction with EMDR: a pilot study. J Psychoactive Drugs. 2014;46(4):303-9.

111 Zweben JE, Clark HW, Smith DE. Traumatic experiences and substance abuse: mapping the territory. J Psychoactive Drugs. 1994;26(4):327-44.

112 Najavits LM, Weiss RD, Shaw SR. The link between substance abuse and posttraumatic stress disorder in women: a research review. Am J Addict. 1997;6(4):273-83.

113 Zweben J, Yeary J. EMDR in the treatment of addiction. J Chem Depend Treat. 2006;8(2):115-27.

114 Carletto S, Oliva F, Barnato M, Antonelli T, Cardia A, Mazzaferro $P$, et al. EMDR as add-on treatment for psychiatric and traumatic symptoms in patients with substance use disorder. Front Psychol. 2018;8:23-33.

115 Bae H, Kim D. Desensitization of triggers and urge reprocessing for an adolescent with internet addiction disorder. J EMDR Pract Res. 2012;6(2):73-81.

116 Ryan ND. Treatment of depression in children and adolescents. Lancet. 2005;366(9489):933-40.

117 Wanders F, Serra M, De Jongh AD. EMDR versus CBT for children with self-esteem and behavioral problems: a randomized controlled trial. J EMDR

Pract Res. 2008;2(3):180-89.

118 Mevissen L, Didden R, Korzilius H, de Jongh A. Eye movement desensitisation and reprocessing therapy for posttraumatic stress disorder in a child and an adolescent with mild to borderline intellectual disability: a multiple baseline across subjects study. J Appl Res Intellect Disabil. 2017;30:34-41.

119 Mevissen L, De Jongh A. PTSD and its treatment in people with intellectual disabilities: a review of the literature. Clin Psychol Rev. 2010;30(3):308-16.

120 Hatton C, Emerson E. The relationship between life events and psychopathology amongst children with intellectual disabilities. J Appl Res Intellect Disabil. 2004;17(2):109-17.

121 Civilotti C, Di Fini G, Acquadro Maran D. Trauma and coping strategies in police officers: a quantitativequalitative pilot study. Int J Environ Res Public Health. 2021;18(3):982.

122 Shapiro F. Handbook of EMDR and family therapy processes. Wiley Online Library; 2007.

123 Pae C-U. Why systematic review rather than narrative review? Psychiatry Investig. 2015;12(3):417-19.

124 Collins JA, Fauser BCJM. Balancing the strengths of systematic and narrative reviews. Oxford: Oxford University Press; 2005.

\section{Publisher's Note}

Springer Nature remains neutral with regard to jurisdictional claims in published maps and institutional affiliations. 